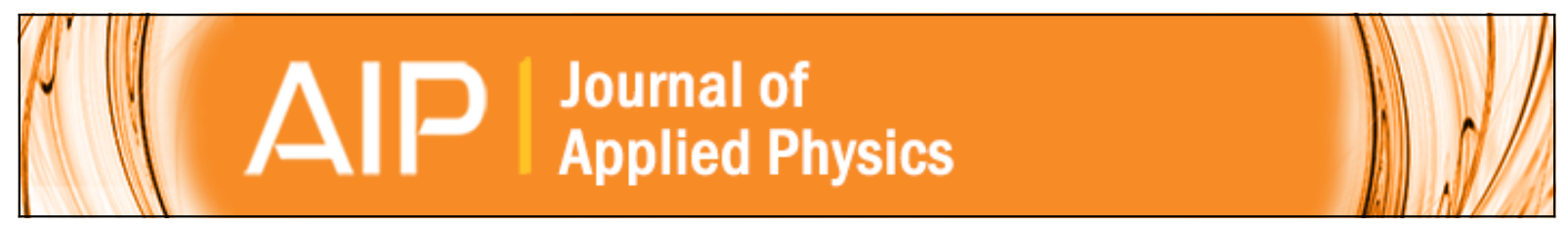

Total secondary-electron yield of metals measured by a dynamic method

Carlos Alberto Fonzar Pintão and Roberto Hessel

Citation: Journal of Applied Physics 88, 478 (2000); doi: 10.1063/1.373682

View online: http://dx.doi.org/10.1063/1.373682

View Table of Contents: http://scitation.aip.org/content/aip/journal/jap/88/1 ?ver=pdfcov

Published by the AIP Publishing

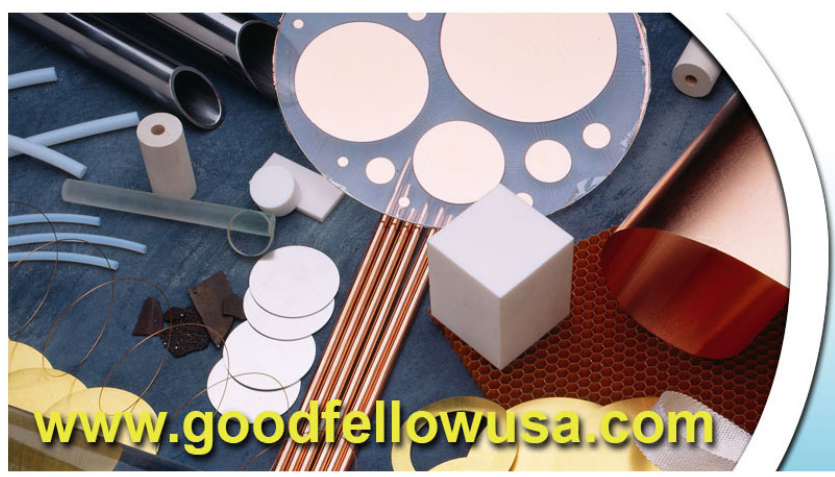

BODIFEllOU metals • ceramics • polymers composites $\cdot$ compounds $\cdot$ glasses

Save $5 \% \cdot$ Buy online 70,000 products $\cdot$ Fast shipping 


\title{
Total secondary-electron yield of metals measured by a dynamic method
}

\author{
Carlos Alberto Fonzar Pintão \\ Universidade Estadual Paulista-UNESP, Departmento de Física, C. P. 473, CEP 17013-360 Bauru, \\ SP, Brazil \\ Roberto Hessel \\ Departamento de Física, Universidade Estadual Paulista-UNESP, C. P. 178, CEP 13500-970 Rio Claro, \\ SP, Brazil
}

(Received 2 November 1998; accepted for publication 28 March 2000)

\begin{abstract}
The secondary electron emission of dielectrics usually is measured by the pulse method, in which the dielectric is irradiated with short pulses of electrons. Attempts to use a dynamic method, in which the dielectric is irradiated continuously, have failed because the dielectric becomes charged and this charge interferes with the emission process. The dynamic method can, however, be applied to metals where volume charges are prevented. This article reports dynamic measurements of the total secondary emission yield from stainless steel, platinum, and aluminum and compares them with results from the current pulse method. In order to apply the dynamic method to metals a simple but important change in the setup was introduced: a dielectric slab was placed between the electrode and the metallic sample, which permitted the sample surface potential and therefore the energy of the incident electrons to change continuously. Unlike for dielectrics, the emission curves for metals are identical when obtained by the two methods. However, for a sample with deliberately oxidized surfaces the total secondary emission yield is smaller when measured with the dynamic method as compared with the pulse method, just as happens for dielectrics. (C) 2000 American Institute of Physics. [S0021-8979(00)03413-7]
\end{abstract}

\section{INTRODUCTION}

When irradiated by an electron beam, solid surfaces emit low-energy secondary and backscattered primary electrons. Numerous authors have investigated this effect and reviews of the problems addressed are found in several books and papers. ${ }^{1,2}$ In order to quantify the emission process it is usual to define the total secondary yield (the ratio between emitted and incident electron number) and study its dependence on the incident beam energy as well as on the properties of the irradiated surface. The total secondary yield can assume values smaller, equal to, or greater than one for irradiation energies below a few $\mathrm{keV}$. The surface of an irradiated dielectric becomes positively or negatively charged as the total yield differs from one. Such polarization can affect the emission process. Most authors have irradiated dielectrics with short electron pulses, while at the same time measuring the electron emission by a separate conveniently poled collector electrode. $^{3-5}$ A new, so-called dynamic method (DM) has been discussed in the literature. ${ }^{6-8}$ This method $(\mathrm{DM})^{8}$ was proposed to study the secondary electron emission from insulators in the planar symmetry: upon charging the insulator surface, the effective energy of the electron beam is changed, and from the knowledge of the beam current, current to the back electrode, and sample capacitance, the whole secondary yield can be obtained in suitably chosen runs. However, as anticipated, the accumulated charges do indeed interfere with the measurement, considerably decreasing the yield. ${ }^{9}$

\footnotetext{
a) Author to whom correspondence should be addressed; electronic mail: fonzar@fc.unesp.br
}

We have found, however, that the DM may be successfully applied to study secondary emission of metals provided a single and obvious change is made: the irradiated metal disk is placed on top of an insulating foil of very small thickness. With this arrangement the metal potential changes during irradiation owing to the rapid dissipation of excess charge to the back surface of the metallic disk facing the insulating foil, therefore avoiding interference between the beam and generated charges. For the sake of comparison measurements were also carried out using (short) electron beam pulses $(\mathrm{PM})^{9}$ without the back insulator foil.

\section{EXPERIMENT}

The setup used, schematically shown in Fig. 1, has already been described in detail. ${ }^{9}$ Its main features are:

(1) the beam energy $E_{P}$ ranges from 0.4 to $20 \mathrm{keV}$, and the current $I_{P}$ from $10^{-6}$ to $10^{-10} \mathrm{~A}$, which is measured by a Faraday cup;

(2) the beam scans an area greater than the sample area, with the sample being $3 \mathrm{~cm}$ in diameter;

(3) the grid-target geometry is plane, with grid transparency of approximately $80 \%$;

(4) a meter measures the current $I_{M}$ to the back electrode which may be grounded or biased to an adjustable potential $V_{2}(0- \pm 3 \mathrm{keV})$;

(5) the back electrode vibrates against the grid (which in our measurements remained grounded) thus allowing the measurement of the potential ${ }^{10}$ of the back electrode by means of a lock-in amplifier (LA) method of the vibrating capacitor; and 


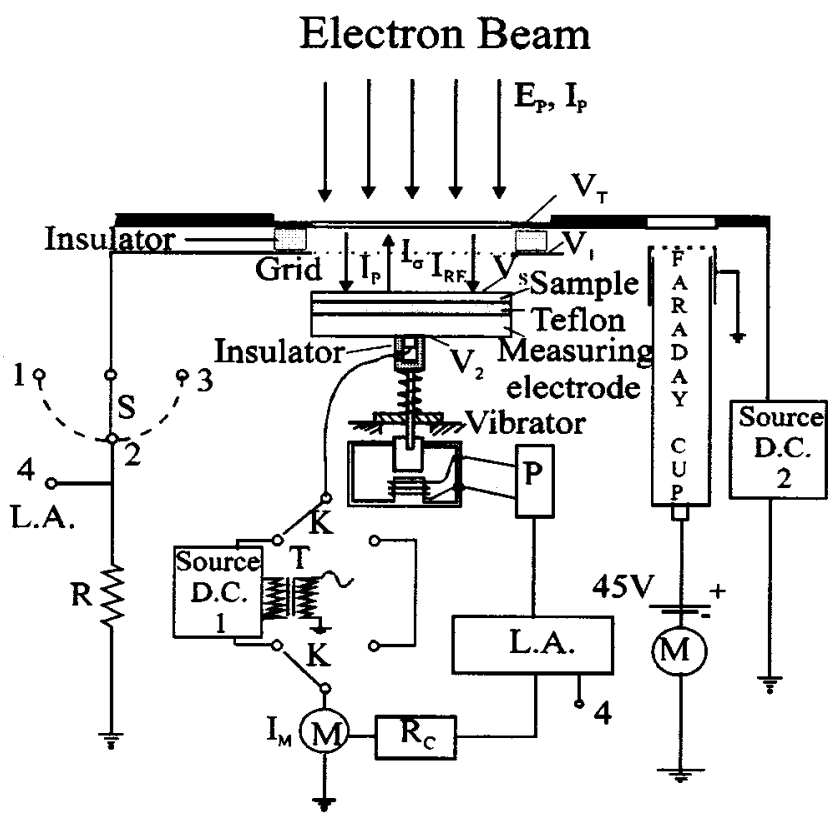

FIG. 1. Scheme of experimental setup. $(M)$ : Keitley meter, $(T)$ : highinsulated coupling capacitor, $(P)$ : signal generator for magnetic coil, $(L A)$ : lock-in amplifier, $(R)$ : measuring resistor for grid signals, $\left(R_{C}\right)$ : double-pen recorder, $\left(V_{S}\right)$ : surface potential, $\left(V_{1}, V_{2}\right.$, and $\left.V_{T}\right)$ : polarization potential for grid, electrode, and cover, respectively; $\left(I_{P}, I_{\sigma}, I_{M}\right.$, and $\left.I_{\mathrm{RE}}\right)$ : current of electrons incident, emitted, measuring, and recaptured, respectively, $\left(E_{P}\right)$ : primary or incident energy of electrons.

(6) the sample is discharged by means of the electron beam under conditions that allow the performance of the "selfregulating mechanism,.6,7

Teflon-FEP ${ }^{\circledR}$ samples $50 \mu \mathrm{m}$ thick, $5 \mathrm{~cm}$ diameter, provided on both faces with a deposited aluminum layer, $3 \mathrm{~cm}$ in diameter, were put directly over the measuring electrode, supporting the metal samples (see Fig. 2). These were $\sim 0.5$ $\mathrm{mm}$ thick polished stainless steel and platinum disks. Measurements were also carried out with the electron beam impinging directly onto the deposited aluminum layer in order to compare the results with those of the same aluminum deposited film exposed deliberately to ambient atmosphere for a long time. A vacuum better than $10^{-4} \mathrm{~Pa}(1$ Torr $=1.33$

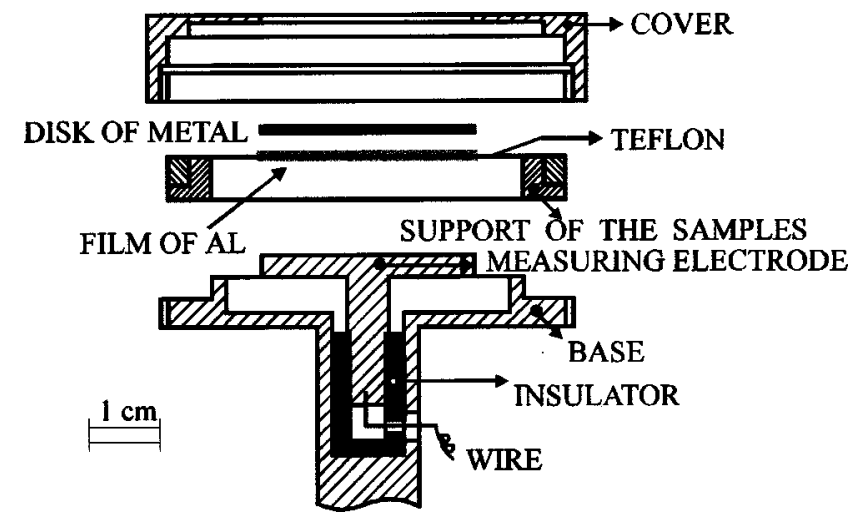

FIG. 2. Support of the samples adapted to apply the dynamic method to metal.

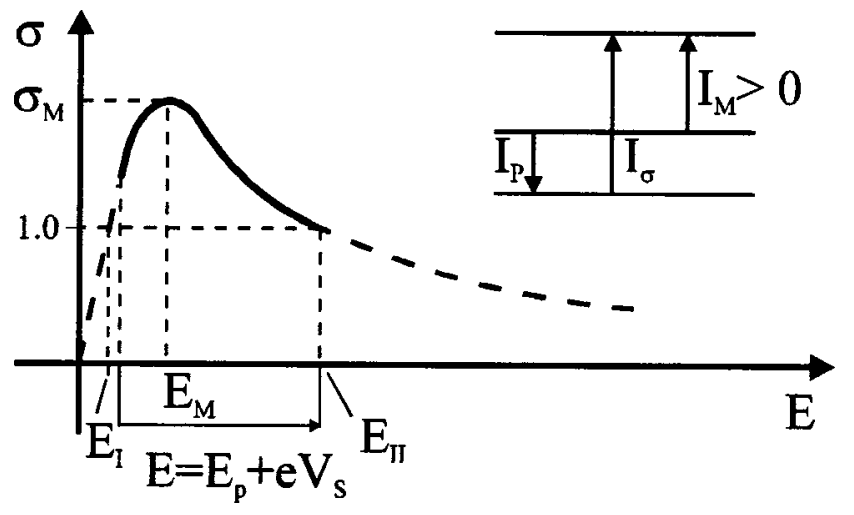

FIG. 3. Total emission yield $\sigma$ as function of energy $E$ (schematic). $E_{\mathrm{I}}$ and $E_{\mathrm{II}}$ are first and second crossover points, $\sigma_{M}$ is maximum yield, and $E_{M}$ energy of maximum yield. The continuous curve represents the section between $E_{\mathrm{I}}$ and $E_{\mathrm{II}}$ obtained from dynamic method. Also shown are vectorial representations of the currents $\left(I_{P}, I_{\sigma}\right.$, and $\left.I_{M}\right)$ indicating the studied interval.

Pa) was used. Our system was not developed to deal with metallic surfaces, for which ultrahigh vacuum is required as in usual surface physics experiments. Therefore, we cannot expect the yields obtained for metals to be comparable with known values in the literature. Our main intent is rather to illustrate the applicability of the dynamic method to metals, which is done as explained later.

\section{A. DM measurements}

In DM measurements we exploit the fact that upon controlling the electron yield $\sigma$, one may charge samples positively or negatively.

(1) Measurement accessing is in the energy range between $E_{\mathrm{I}}$ and $E_{\mathrm{II}}$, the first and second crossover points, respectively. The beam energy is somewhat larger than $E_{\mathrm{II}}$ and the bias voltage $V_{2}$ so chosen that the effective initial energy $E_{i}$ is a little higher than $E_{\mathrm{I}}$. For this $V_{2}$ is made negative and such that $-e V_{2}<E_{p}-E_{\mathrm{I}}, e$ being the electronic charge. The sample is charged positively, and its potential increases by $V_{S}$ causing the effective energy $E$ to be equal to $E_{P}$ $+e V_{S}$. Finally the second crossover point $E_{\mathrm{II}}$ is reached, when charging ends. This is shown in Fig. 3, where $E_{M}$ is the energy for maximum yield. The insert shows the current balance $I_{\sigma}$ being the secondary current define as $I_{\sigma}=I_{M}$ $-I_{P}$, with $I_{\mathrm{RE}}=0$. The yield $\sigma$ is calculated from

$$
\sigma=-\frac{I_{\sigma}}{I_{P}}=1-\frac{I_{M}}{I_{P}}
$$

where the currents are considered negative downward (therefore $I_{M}$ in Fig. 3 is positive).

(2) Measurement accessing energies below $E_{\mathrm{I}}$ and above $E_{\mathrm{II}}$. In these cases $V_{2}$ is made zero and $E_{P}$ is chosen, respectively: (a) well below $E_{\mathrm{I}}$, with charging finishing when $V_{S}$ reaches $V_{F}$ (filament potential); (b) well above $E_{\mathrm{II}}$, with charging finishing when $E=E_{P}+e V_{S}$ becomes equal to $E_{\mathrm{II}}$. This is shown in Fig. 4. 


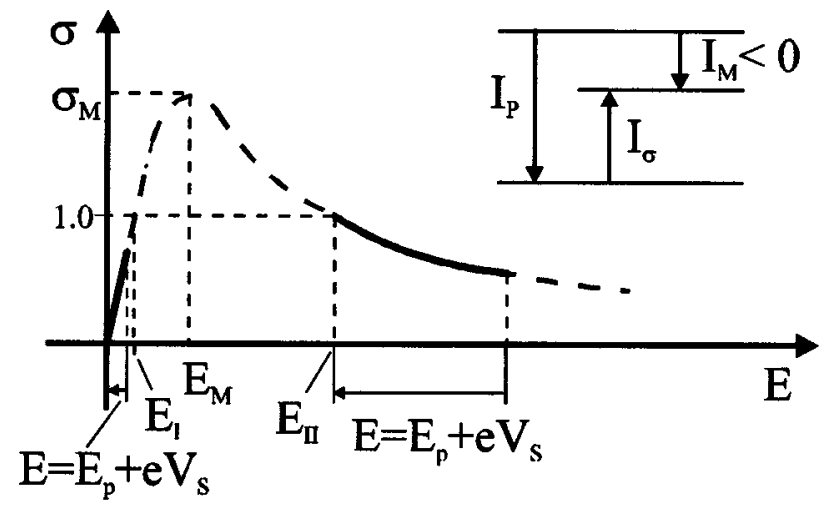

FIG. 4. $\sigma$ as function of energy $E$ (schematic). The continuous curve represent the sections above $E_{\mathrm{II}}$ and below $E_{\mathrm{I}}$ obtained from dynamic method. Also shown are vectorial representations of the currents $\left(I_{P}, I_{\sigma}\right.$, and $\left.I_{M}\right)$ indicating the interval studied.

\section{B. PM measurements}

Measurements with short pulses (the pulse duration is not important) and with the metal disk functioning as the back electrode were carried out for comparison with DM results. In this method the beam energy $E_{P}$ is fixed and the electrode potential $V_{2}$ is adjusted, step by step, in order to cover the desired range of energies.

\section{RESULTS AND DISCUSSION}

In Figs. 5-7 we show the yield $\sigma$ as a function of the effective beam energy $E$ for a stainless steel disk, a platinum disk, and an aluminum deposited film for a beam current of $2.25 \mathrm{nA}$, but the results are essentially independent of this parameter. In each case, the results obtained with the PM are compared with those with the DM starting at different initial effective energies $E_{i}$, either to cover the desired range of energies, as explained in Sec. II A, or for the sake of illus-

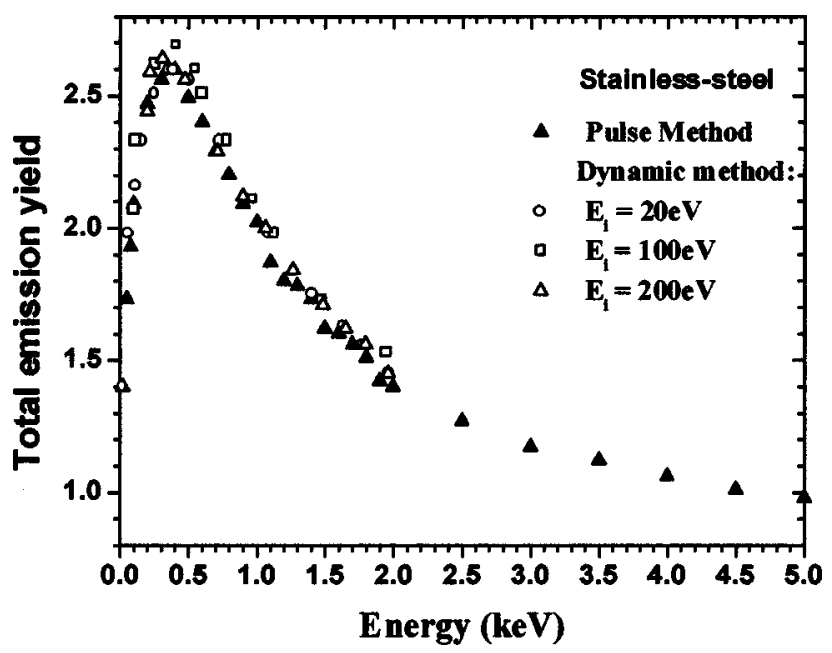

FIG. 5. Total emission yield as a function of energy for a $0.5 \mathrm{~mm}$ stainless steel disk. Pulse method: section between $E_{\mathrm{I}}=0.020 \mathrm{keV}$ and $E_{\mathrm{II}}=4.75$ $\mathrm{keV}, E_{p}=2.0 \mathrm{keV}, 4.0 \mathrm{keV}, V_{2}$ was selected from -100 to $-2100 \mathrm{~V}$. Section above $E_{\mathrm{II}}, E_{p}=6.0 \mathrm{keV}, V_{2}=-1000 \mathrm{~V}$. Dynamic method: section between $E_{\mathrm{I}}$ and $E_{\mathrm{II}}, E_{p}=2.0 \mathrm{keV}, V_{2}$ was selected in $-1980,-1900$, and $-1800 \mathrm{~V}$. In all cases $I_{P}$ was $2.25 \mathrm{nA}$.

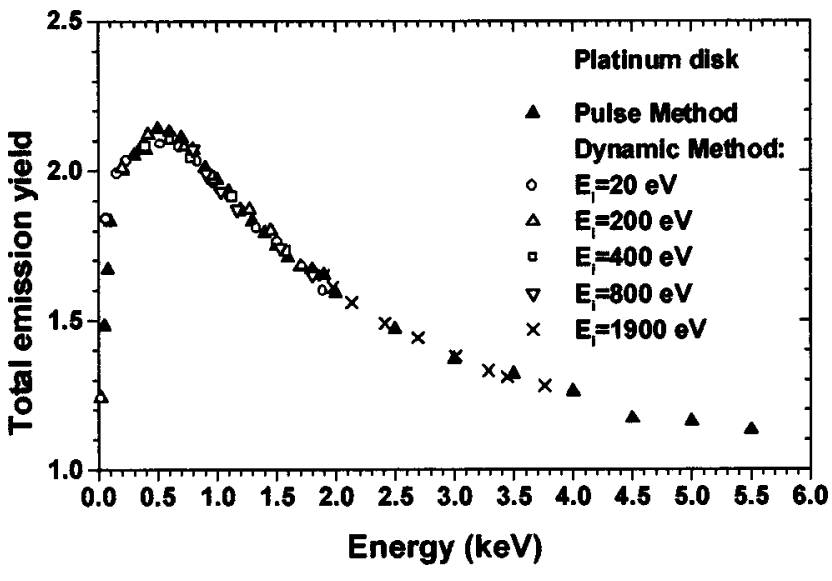

FIG. 6. Total emission yield as a function of energy for a $0.2 \mathrm{~mm}$ platinum disk. Pulse method: section between $E_{\mathrm{I}}=0.020 \mathrm{keV}$ and $E_{\mathrm{II}}=7.0 \mathrm{keV}, E_{p}$ $=2.0 \mathrm{keV}, V_{2}$ was selected from -200 to $-1980 \mathrm{~V}$. $E_{p}=4.0 \mathrm{keV}, V_{2}$ was selected from -500 to $-2100 \mathrm{~V}$ and $E_{P}=6.0 \mathrm{keV}, V_{2}$ was selected from -500 to $-2000 \mathrm{~V}$. Dynamic method: section between $E_{\mathrm{I}}$ and $E_{\mathrm{II}}, E_{p}$ $=2.0 \mathrm{keV}, V_{2}$ was selected in $-1980,-1800,-1600$, and $-1200 \mathrm{~V}$ and $E_{p}=4.0 \mathrm{keV}, V_{2}$ was selected in $-2100 \mathrm{~V} ; I_{P}=2.25 \mathrm{nA}$.

trating the reliability of the method. In all cases the curves are reasonably well superimposed on each other. This behavior indicates that possible impurities (such as an oxide layer on aluminum and platinum disks), which should expect to be present since ultrahigh vacuum was not employed, do not affect the conductivity of the surface, at least as far as electron emission is concerned. For insulating materials, the two methods indeed lead to different results. For instance, Hessel and Gross ${ }^{11}$ showed that for polymers $\sigma$ obtained with DM are smaller than with PM and depend on $E_{i}$, which indicated that charge accumulation over the polymer surface impairs the use of the DM method for insulating materials. Therefore, even if oxide layers were formed on the metal plates, they probably are unable to store considerable amounts of charges, as explained below.

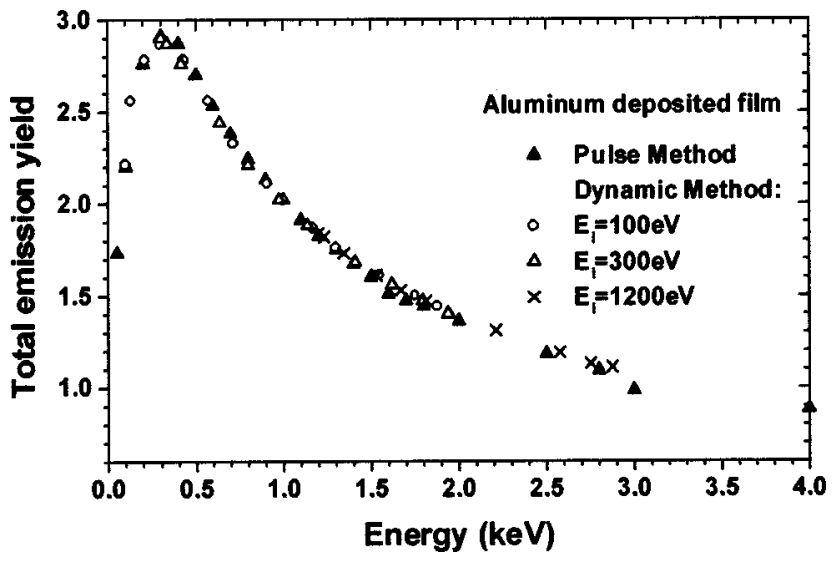

FIG. 7. Total emission yield as a function of energy for a $100 \mathrm{~nm}$ aluminum deposited film. Pulse method: section between $E_{\mathrm{I}}=0.020 \mathrm{keV}$ and $E_{\mathrm{II}}$ $=3.0 \mathrm{keV}, E_{p}=2.0 \mathrm{keV}, V_{2}$ was selected from -200 to $-1950 \mathrm{~V}, E_{p}$ $=3.0 \mathrm{keV}, V_{2}$ was selected from -200 to $-1500 \mathrm{~V}$. Section above $E_{\mathrm{II}}, E_{p}=4.5 \mathrm{keV}, V_{2}$ was selected from -500 to $-1500 \mathrm{~V}$. Dynamic method: section between $E_{\mathrm{I}}$ and $E_{\mathrm{II}}, E_{p}=2.0 \mathrm{keV}, V_{2}$ was selected in $-1900 \mathrm{~V},-1700 \mathrm{~V}, E_{p}=3.0 \mathrm{keV}, V_{2}$ was selected in $-1800 ; I_{P}=2.25 \mathrm{nA}$. 


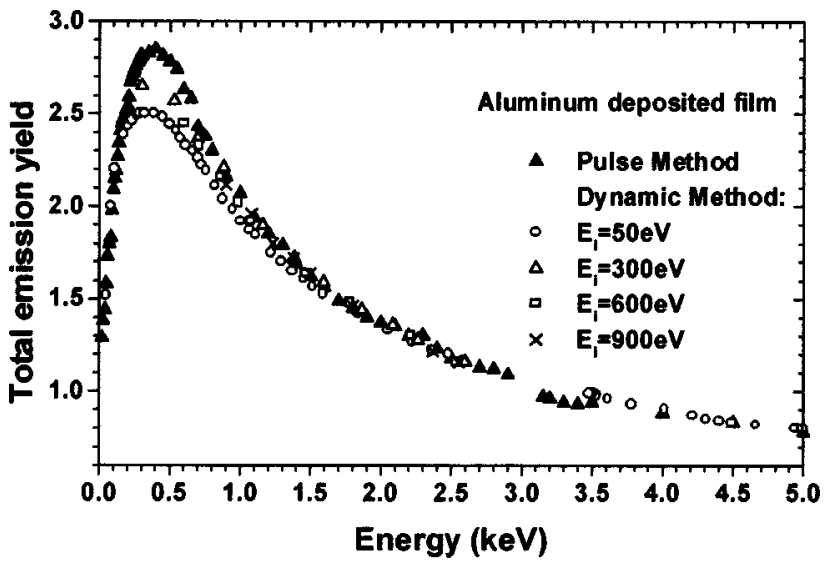

FIG. 8. Total emission yield as a function of energy for a $100 \mathrm{~nm}$ aluminum deposited film that was exposed deliberately to the atmosphere. Pulse method: section between $E_{\mathrm{I}}=0.015 \mathrm{keV}$ and $E_{\mathrm{II}}=3.1 \mathrm{keV}, E_{p}=2.0 \mathrm{keV}, V_{2}$ was selected from -200 to $-1980 \mathrm{~V}, E_{p}=3.0 \mathrm{keV}, V_{2}$ was selected from -200 to $-1200 \mathrm{~V}$. Section above $E_{\mathrm{II}}, E_{p}=5.5 \mathrm{keV}, V_{2}$ was selected from -500 to $-2400 \mathrm{~V}$. Dynamic method: section between $E_{\mathrm{I}}$ and $E_{\mathrm{II}}, E_{p}$ $=2.7 \mathrm{keV}, V_{2}$ was selected in $-2650,-2400,-2100$, and $-1800 \mathrm{~V}, E_{p}$ $=5.2 \mathrm{keV}, V_{2}$ was selected in $-200 \mathrm{~V} ; I_{P}=2.25 \mathrm{nA}$.

In a subsidiary experiment, the aluminum deposited film was exposed to ambient atmosphere for a long period of time. As expected an oxide layer developed, which now affected the yield $\sigma$ considerably. The positive charge stored in the aluminum oxide layer prevent some low energy electrons ("true" secondary electrons) from escaping from the surface, thus leading to a smaller $\sigma$ in the DM method, as shown in Fig. 8. It is concluded that charges created during the emission process suffer much faster recombination in metals than in insulating materials, and therefore they do not modify $\sigma$. The fact that emission curves $\sigma \times E$ obtained from both methods are the same and the fact that $\sigma$ does not depend on the energy $E_{i}$ of the impinging electrons show that the depth at which charges are created within the sample or their mobility does not affect the results, unlike in polymers. ${ }^{11}$ This means that the charge recombination process, which gives origin to low energy electrons, is sufficiently fast not to interfere with the electrons that are emitted by the sample surface.

In summary, upon using the DM and PM methods independently, the sample behavior can be classified as that of a metal or insulator during the emission process. In order to reinforce this claim, we analyzed some of the results quantitatively. It is known that for energies much larger than $E_{M}$, the secondary emission yield $\delta$ is proportional $E^{-n}$, where $n=N-1$. For metals $N$ is 1.35 according to Young ${ }^{12}$ or 1.66 according to Lane and Zaffarano, ${ }^{13}$ while in polymers (insulating) Matskevich ${ }^{14}$ and Burke ${ }^{15}$ quoted $N$ as approximately 1.72 . The secondary emission yield $\delta$, which we also determined for a platinum disk and Teflon-FEP ${ }^{\circledR}$, introducing some modifications in the measuring system, ${ }^{16}$ is equal to $\sigma-\eta$, where $\eta$ is the backscattering emission yield. In the double logarithmic scale the yield curves should therefore be given by straight lines. Figure 9 shows $\log \delta$ vs $\log E$, from which we obtained that $n=0.50$ for a platinum disk, which is closer to the value expected for metals than $n=0.70$ for Teflon-FEP ${ }^{\circledR}$. Obviously a precise determination of such a

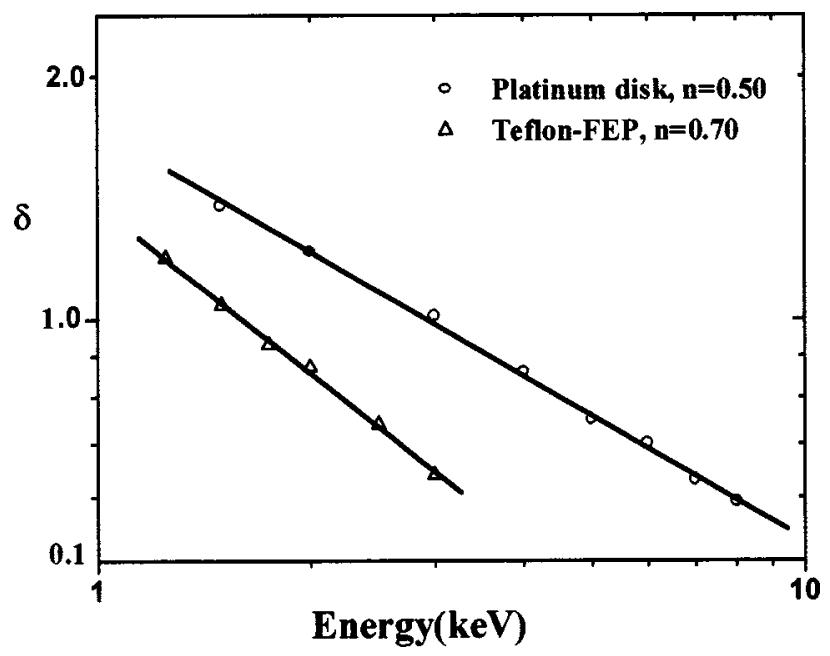

FIG. 9. High energy section of secondary electron emission curves in double logarithmic scale, confirming values of $n$ for metals $(0.35-0.66)$ and insulators $(\approx 0.72)$.

parameter for metals should use data obtained at ultrahigh vacuum, but the feasibility of the DM method has nevertheless been demonstrated.

\section{CONCLUSION}

Using a dielectric between the electrode and the studied sample it is possible to extend the dynamic method to metals. A new result obtained with a sample of a stainless-steel disk shows that the dynamic method works for the metals and that, if appropriate technical changes could be made, our apparatus would provide results similar to those obtained under ultrahigh vacuum conditions. Other samples such as a platinum disk and an aluminum deposited film (compatible with high vacuum conditions) show identical values for total secondary emission yields when the dynamic and current pulse methods are applied. We propose that the similarity observed for metals is due to the fact that charges generated during the emission and irradiation process do not interfere with the value of the emission currents because they recombine quickly, independently of the depth in which they are created, their amount, and their mobility. The different behavior observed for a sample of aluminum deposited film that was exposed deliberately to the atmosphere for a long time is due to the charges generated during the irradiation that do interfere with the values of the emission currents.

\section{ACKNOWLEDGMENTS}

The authors would like to thank CNPq and FAPESP for financial support, G. F. L. Ferreira for his encouragement, Dr. O. N. Oliveira, Jr., for proofreading the manuscript, and Professor Bernhard Gross (advisor for both authors in their Ph.D work), for reading, corrections, and suggestions.

${ }^{1}$ J. Dekker, in Solid State Physics, edited by F. Seitz and D. Turnbull (Academic, New York, 1958), Vol. 6, pp. 251-315.

${ }^{2}$ H. Seiler, J. Appl. Phys. 54, R1 (1983).

${ }^{3}$ H. L. Heydt, Rev. Sci. Instrum. 21, 639 (1950).

${ }^{4}$ J. B. Johnson and M. G. McKay, Phys. Rev. 91, 582 (1953).

${ }^{5}$ E. J. Sternglass, Phys. Rev. 95, 345 (1954). 
${ }^{6}$ B. Gross, H. Von Seggern, and T. E. West, J. Appl. Phys. 56, 2333 (1984).

${ }^{7}$ B. Gross, H. VonSeggern, and H. Berraissoul, IEEE Trans. Electr. Insul. 22, 23 (1987).

${ }^{8}$ H. Von Seggern, IEEE Trans. Nucl. Sci. NS-32, 1503 (1985).

${ }^{9}$ B. Gross and R. Hessel, IEEE Trans. Electr. Insul. 26, 18 (1991).

${ }^{10}$ R. A. Moreno and B. Gross, J. Appl. Phys. 47, 3397 (1976).
${ }^{11}$ R. Hessel and B. Gross, IEEE Trans. Electr. Insul. 27, 741 (1992).

${ }^{12}$ J. R. Young, Phys. Rev. 103, 292 (1956).

${ }^{13}$ R. O. Lane and D. I. Zaffarano, Phys. Rev. 94, 960 (1954).

${ }^{14}$ T. L. Matskevich, Fiz. Tverd. Tela Akad. Nauk. SSSR 1, 277 (1959).

${ }^{15}$ E. A. Burke, IEEE Trans. Nucl. Sci. NS-27, 1760 (1980).

${ }^{16}$ C. A. F. Pintão, Ph.D thesis, São Carlos: USP-IFSC, 1996. 